\title{
Systematic study of thermal stability of AIGaN/GaN two-dimensional electron gas structure with SiN surface passivation
}

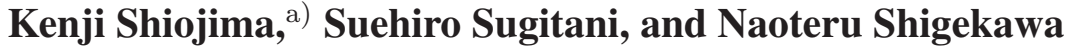 \\ NTT Photonics Laboratories, Nippon Telegraph and Telephone Corporation. \\ 3-1 Wakamiya, Morinosato, Atsugi, Kanagawa 243-0199, Japan \\ a) shiojima@aecl.ntt.co.jp
}

\begin{abstract}
An annealing study of the AlGaN/GaN 2DEG structure for HEMTs with or without SiN surface passivation films was conducted with the AlGaN layer thickness dependence taken into consideration. Without SiN, the sheet resistance of the samples with thin AlGaN layers increased significantly upon annealing at 620 and $800^{\circ} \mathrm{C}$. In contrast, samples with SiN were thermally stable after annealing at up to $800^{\circ} \mathrm{C}$ even when the AlGaN layer was as thin as $152 \AA$. TEM observations revealed that neither surface roughness nor interfacial diffusion at the SiN/AlGaN interface occurred due to annealing. The SiN layer is very effective for passivating an AlGaN surface and improving the thermal stability of a thin-AlGaN 2DEG channel.
\end{abstract}

Keywords: AlGaN/GaN heterostructure, SiN surface passivation, sheet resistance, thermal stability, AlGaN thickness dependence

Classification: Electron devices

\section{References}

[1] B. E. Foutz, L. F. Eastman, U. V. Bhapkar, and M. S. Shur, "Comparison of high field electron transport in GaN and GaAs," Appl. Phys. Lett., vol. 70, pp. 2849-2851, 1997.

[2] Y. -F. Wu, B. P. Keller, S. Keller, D. Kapolnek, S. P. DenBaars, and U. K. Mishra, "Measured microwave power performance of AlGaN/GaN MODFET," IEEE Electron Device Lett., vol. 17, pp. 455-457, 1996.

[3] W. Lu, J. Yang, M. A. Khan, and I. Adesida, "AlGaN/GaN HEMTs on $\mathrm{SiC}$ with over $100 \mathrm{GHz}_{\mathrm{T}}$ and low microwave noise," IEEE Trans. Electron Devices, vol. 48, pp. 581-585, 2001.

[4] J. H. Edgar, S. Strite, I. Akasaki, H. Amano, and C. Wetzel, Properties, Processing and Applications of Gallium Nitride and Related Semiconductors, p. 491, INSPEC publication, London, 1999.

[5] K. Shiojima and N. Shigekawa, "Thermal Stability of Sheet Resistance in AlGaN/GaN 2DEG Structure," Phys. Status Solidi C, vol. 0, pp. 397-400, 2002.

[6] K. Shiojima and N. Shigekawa, "Thermal Stability of Electrical Properties in AlGaN/GaN Heterostructures," Jpn. J. Appl. Phys., vol. 43, pp. 100- 
$105,2004$.

[7] M. M. Sung, J. Ahn, V. Bykov, J. W. Rabalais, D. D. Koleske, and A. E. Wichenden, "Composition and structure of the GaN\{000-1\}-(1x1) surface," Phys. Rev. B, vol. 54, pp. 14652-14669, 1996.

[8] T. Hashizume, S. Ootomo, S. Oyama, M. Konishi, and H. Hasegawa, "Chemistry and electrical properties of surfaces of GaN and GaN/AlGaN heterostructures," J. Vac. Sci. Tecnol. B, vol. 19(4), pp. 1675-1681, 2001.

[9] X. Hu, A. Koudymov, G. Simin, J. Yang, M. Asif Khan, A. Tarakji, M. S. Shur, and R. Gaska, " $\mathrm{SiN}_{3} \mathrm{~N}_{4} / \mathrm{AlGaN} / \mathrm{GaN}$-metal-insulatorsemiconductor heterostructure field-effect transistors," Appl. Phys. Lett., vol. 79 , pp. $2832-2834,2001$.

\section{Introduction}

AlGaN/GaN high-electron-mobility transistors (HEMT) have been intensively studied as high-temperature high-power high-frequency electron devices because GaN has a higher breakdown field and larger saturation velocity than GaAs and also exhibits excellent transport properties under a high electric field $(>100 \mathrm{kV} / \mathrm{cm})[1]$. One of the main applications of AlGaN/GaN HEMTs is in the power amplifiers of base stations for wireless communication. This application requires 100-W-class output power in the L-band, and submicrometer-gate AlGaN/GaN HEMTs whose typical device parameters are AlGaN thickness of $\sim 300 \AA$, Al content of $\sim 25 \%$, sheet carrier density $\left(\mathrm{n}_{\mathrm{s}}\right)$ of $\sim 1 \times 10^{13} \mathrm{~cm}^{-2}$ have been demonstrated [2]. On the other hand, by scaling down device dimensions, i.e., reducing gate length $\left(\mathrm{L}_{\mathrm{g}}\right)$ to around $0.1 \mu \mathrm{m}, \mathrm{RF}$ performance of over a $100-\mathrm{GHz}$ cutoff frequency $\left(\mathrm{f}_{\mathrm{T}}\right)$ and maximum oscillation frequency $\left(f_{\max }\right)$ have been achieved [3]. A HEMT structure with a shorter $\mathrm{L}_{\mathrm{g}}(<0.1 \mu \mathrm{m})$, shallower channel (AlGaN thickness of $200 \AA$ ), and larger mobility $(\mu) \cdot \mathrm{n}_{\mathrm{s}}$ product is required for further improvement of $\mathrm{RF}$ performance for millimeter-wave applications. In the actual field effect transistor (FET) process, a high-temperature anneal $\left(\sim 800^{\circ} \mathrm{C}\right)$ is required in order to form $\mathrm{Ti} / \mathrm{Al}$ ohmic contacts [4]. Hence, the thermal stabilities of the thin AlGaN layer and two-dimensional electron gas (2DEG) structure are also important.

We conducted an annealing study of $\mathrm{AlGaN} / \mathrm{GaN}$ heterostructure wafers for HEMTs and found that the sheet resistance $\left(\mathrm{R}_{\text {sheet }}\right)$ increases when annealing is performed below the growth temperature $\left(\sim 1000^{\circ} \mathrm{C}\right)$ and that the increase depends on the AlGaN crystal quality and thickness, especially at thicknesses less than $200 \AA[5,6]$. The origin of the $\mathrm{R}_{\text {sheet }}$ increase upon annealing would be in the vicinity of the AlGaN surface and caused by surface contamination with $\mathrm{O}$ and $\mathrm{C}$ atoms [6] and $\mathrm{N}$ outdiffusion [7]. To suppress the increase of $\mathrm{R}_{\text {sheet }}$, we used SiN surface passivation films on the AlGaN layers. Because SiN forms a low-interfacial-state-density interface to GaN and AlGaN [8], SiN films are used as the surface passivation layer of AlGaN/GaN HEMTs to reduce current collapse and as an insulator in the metal-insulatorsemiconductor structure [9]. However, the effect of the SiN passivation on 
the thermal stability of the AlGaN/GaN 2DEG channel has not been well investigated.

This paper reports a systematic study of the thermal stability of the AlGaN/GaN 2DEG structure with or without SiN passivation layers in conjunction with actually measured AlGaN layer thickness.

\section{Samples and experimental methods}

Figure 1 shows the cross-sectional epitaxial structure, which was grown on a two-inch (0001)-plane sapphire substrate by metal-organic chemical vapor deposition. The structure comprises a semi-insulating GaN layer $(3 \mu \mathrm{m})$, an undoped- $\mathrm{Al}_{0.25} \mathrm{Ga}_{0.75} \mathrm{~N}$ spacer layer, an $\mathrm{n}-\mathrm{Al}_{0.25} \mathrm{Ga}_{0.75} \mathrm{~N}$ carrier-supply layer (Si doping concentration of $1 \times 10^{19} \mathrm{~cm}^{-3}$ ), and an undoped $\mathrm{Al}_{0.25} \mathrm{Ga}_{0.75} \mathrm{~N}$ cap layer. The thickness of each AlGaN layer was assigned with a fixed ratio for all samples (undoped AlGaN spacer:n-AlGaN carrier supply layer:undoped AlGaN cap $=17: 55: 28)$. Three different-total-AlGaN-thickness wafers were grown. The total AlGaN layer thickness was actually determined by grazing incidence X-ray reflectivity measurements because determining AlGaN thickness from the epitaxial growth rate is less reliable. The measured thicknesses were 207, 161, and $152 \AA$. Eddy current measurements were repeatedly conducted to characterize the $\mathrm{R}_{\text {sheet }}$ nondestructively at the same point before and after annealing. The $\mathrm{R}_{\text {sheet }}$ values of each wafer ranged from 543 to $612 \Omega /$ sq in the as-grown state.

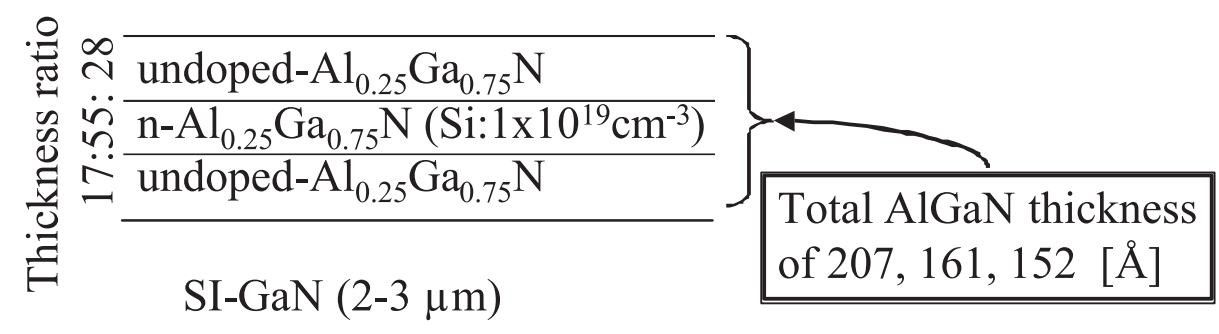

sapphire sub.

Fig. 1. Schematic cross section of the AlGaN/GaN 2DEG epitaxial structure. Three samples with different AlGaN thicknesses were grown. The thickness of each AlGaN layer was assigned with a fixed ratio for all samples.

Firstly, the two-inch GaN wafers were loaded into a plasma-assisted chemical vapor deposition (p-CVD) chamber. Half of each wafer was covered with a glass plate to prevent $\mathrm{SiN}$ deposition. The SiN films were deposited on the other half using silane and ammonia gases. This method eliminates waferto-wafer variations. Then, the whole wafer was subjected to isochronal rapid thermal annealing (RTA) at 620 and $800^{\circ} \mathrm{C}$ for $30 \mathrm{~s}$ in $99.9999 \% \mathrm{~N}_{2}$ flow. After the annealing, the SiN/AlGaN interface was observed by cross-sectional transmission electron microscopy (TEM). 


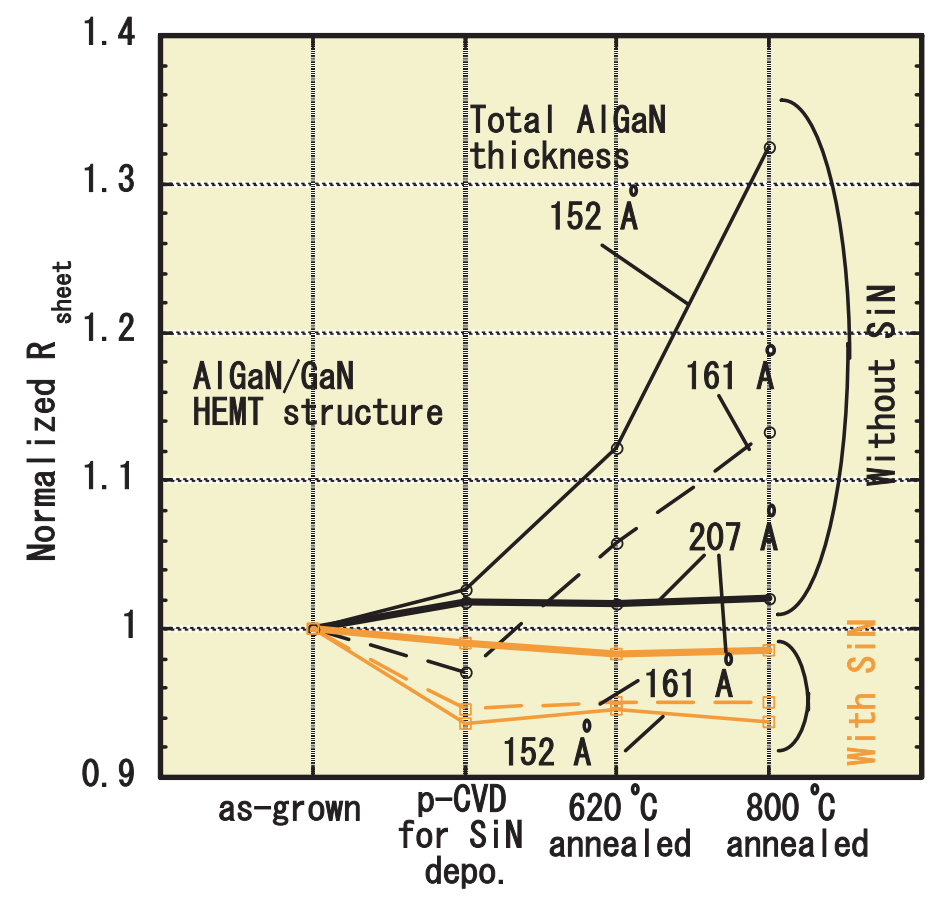

Fig. 2. Variation of $\mathrm{R}_{\text {sheet }}$ upon annealing for the samples with or without the SiN surface passivation films.

\section{Results and discussion}

Figure 2 shows the $\mathrm{R}_{\text {sheet }}$ normalized by the as-grown value after the $\mathrm{SiN}$ deposition and 620 and $800^{\circ} \mathrm{C}$ annealing for three different-AlGaN-thickness samples with or without the SiN surface passivation films. For the samples without SiN, after the p-CVD process, a small variation within $\pm 2 \%$ was seen. As mentioned in the previous section, the wafers were in the p-CVD ambient, but essentially the AlGaN surfaces were not exposed to the plasma. This small variation may be attributable to the unintentional exposure of the AlGaN surface to the silane and ammonia gases like in a gas-phase surface treatment. The $R_{\text {sheet }}$ of the $207-\AA$-thick sample was stable even after the $800^{\circ} \mathrm{C}$ annealing. Meanwhile, the $161-\AA$-thick sample showed $\mathrm{R}_{\text {sheet }}$ increases of 6.1 and $13.7 \%$ after the 620 and $800^{\circ} \mathrm{C}$ annealing, respectively. The 152 - $\AA$-thick sample showed an $\mathrm{R}_{\text {sheet }}$ increase of $33 \%$ after the $800^{\circ} \mathrm{C}$ annealing. These results are consistent with our previous finding, that an AlGaN/GaN HEMT structure with a less-than-200- $\AA$-thick AlGaN layer is thermally unstable [6]. In contrast to the unpassivated samples, the samples with SiN were very stable on annealing. After the SiN deposition, the $\mathrm{R}_{\text {sheet }}$ of the 207 - $\AA$-thick sample decreased only $1 \%$, and that of the 161 - and 152 - $\AA$-thick samples decreased about $6 \%$. These small $R_{\text {sheet }}$ decreases were normally observed in our experimental conditions. After removing the SiN with a chemical solution, the $R_{\text {sheet }}$ returned to the as-grown value. This indicates that process-induced damages were not responsible. A possible explanation is downward band bending due to the SiN deposition. The $\mathrm{R}_{\text {sheet }}$ values of all the passivated samples were stable within $1 \%$ fluctuation up to $800^{\circ} \mathrm{C}$ annealing. Therefore, the SiN surface passivation is effective way to 
prevent $\mathrm{R}_{\text {sheet }}$ increasing upon annealing even when the AlGaN layer is as thin as $152 \AA$.

Figure 3 shows the cross-sectional TEM image of the sample after $620^{\circ} \mathrm{C}$ annealing at $900000 x$ magnification. The $\mathrm{SiN} / \mathrm{AlGaN}$ interface can be clearly seen and was atomically flat. Neither surface roughness nor interfacial diffusion was observed. The SiN layer was still amorphous after the annealing. The improved thermal stability for a thin-AlGaN 2DEG channel was thusly also confirmed from the structural point of view.

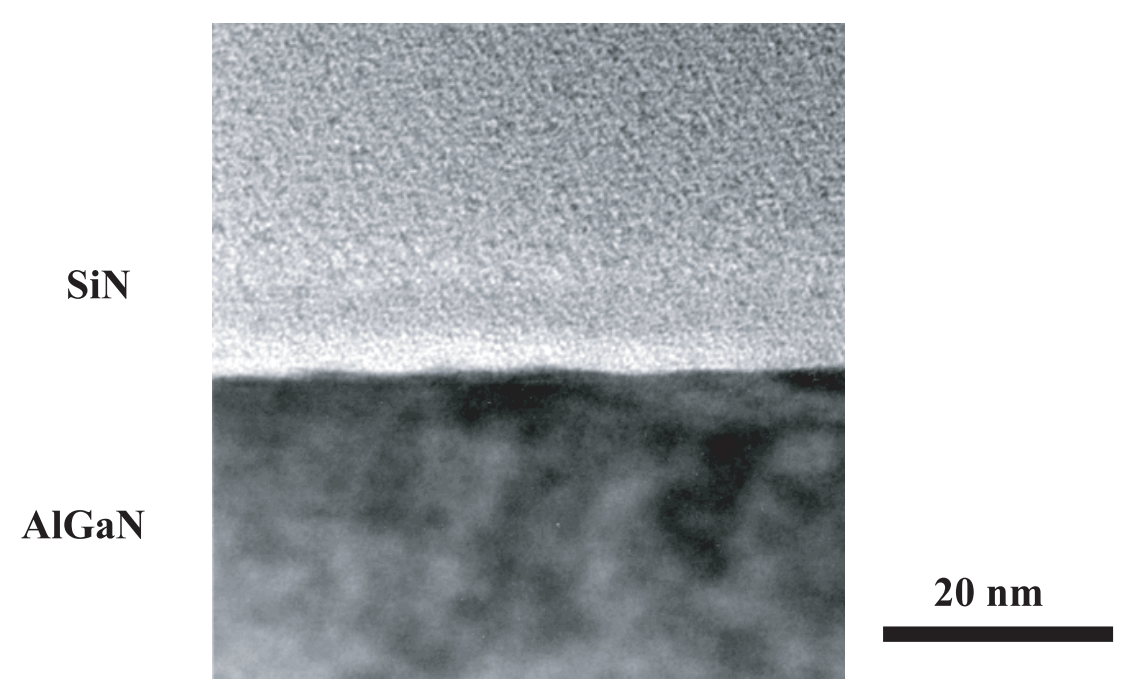

Fig. 3. Cross-sectional TEM image of the sample after $620^{\circ} \mathrm{C}$ annealing.

\section{Conclusion}

An annealing study of the AlGaN/GaN 2DEG structure for HEMTs with or without SiN surface passivation films was conducted with the AlGaN layer thickness dependence taken into consideration. Without the SiN layers, the $\mathrm{R}_{\text {sheet }}$ of samples with 161 and $152 \AA$-thick AlGaN layers increased significantly when annealing was performed at 620 and $800^{\circ} \mathrm{C}$. In contrast, samples with the SiN were thermally stable after annealing at up to $800^{\circ} \mathrm{C}$, even when the AlGaN layer was as thin as $152 \AA$. TEM observations revealed that the $\mathrm{SiN} / \mathrm{AlGaN}$ interface was atomically flat, and neither surface roughness nor interfacial diffusion was observed. Based on these results, we can conclude that the SiN layer is very effective for passivating an AlGaN surface and forming a thermally-stable thin-AlGaN 2DEG channel.

\section{Acknowledgments}

The authors thank Drs. H. Toba, and M. Muraguchi of NTT Photonics Laboratories for their encouragement. 